\title{
A cost comparison of rasburicase versus dialysis in the management of children with acute leukaemia and lymphoma at a South African centre
}

\begin{tabular}{|c|c|}
\hline $\begin{array}{l}\text { Authors: } \\
\text { Morné F. Kah } \\
\text { Alan Davidsor } \\
\text { Ann van Eysse } \\
\text { Peter Nourse } \\
\text { Mignon McC } \\
\text { Marc Hendric }\end{array}$ & $\begin{array}{l}\mathrm{s}^{1} \mathbb{1} \\
2 \mathbb{1} \\
\mathrm{n}^{2} \mathbb{0} \\
\mathbb{1 0} \\
\mathrm{lloch}^{2} \mathbb{0} \\
\mathrm{ss}^{2} \mathbb{0}\end{array}$ \\
\hline \multicolumn{2}{|c|}{$\begin{array}{l}\text { Affiliations: } \\
{ }^{1} \text { Groote Schuur Hospital, } \\
\text { Cape Town, South Africa }\end{array}$} \\
\hline \multicolumn{2}{|c|}{$\begin{array}{l}{ }^{2} \text { Red Cross War Memorial } \\
\text { Children's Hospital, Cape } \\
\text { Town, South Africa }\end{array}$} \\
\hline \multicolumn{2}{|c|}{$\begin{array}{l}\text { Corresponding author: } \\
\text { Morne Kahts, } \\
\text { morne@kahts.net }\end{array}$} \\
\hline \multicolumn{2}{|c|}{$\begin{array}{l}\text { Received: } 18 \text { July } 2018 \\
\text { Accepted: } 02 \text { Oct. } 2018 \\
\text { Published: } 29 \text { Oct. } 2018\end{array}$} \\
\hline \multicolumn{2}{|c|}{$\begin{array}{l}\text { How to cite this article: } \\
\text { Kahts MF, Davidson A, } \\
\text { Van Eyssen A, Nourse P, } \\
\text { McCulloch M, Hendricks M. } \\
\text { A cost comparison of } \\
\text { rasburicase versus dialysis in } \\
\text { the management of children } \\
\text { with acute leukaemia and } \\
\text { lymphoma at a South African } \\
\text { centre. S. Afr. j. oncol. 2018; } \\
\text { 2(0), a50. https://doi.org/ } \\
\text { 10.4102/sajo.v2i0.50 }\end{array}$} \\
\hline \multicolumn{2}{|c|}{$\begin{array}{l}\text { Copyright: } \\
\text { (c) 2018. The Authors. } \\
\text { Licensee: AOSIS. This wo } \\
\text { is licensed under the } \\
\text { Creative Commons } \\
\text { Attribution License. }\end{array}$} \\
\hline \multicolumn{2}{|c|}{ Read online: } \\
\hline 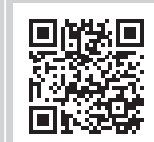 & $\begin{array}{l}\text { Scan this QR } \\
\text { code with your } \\
\text { smart phone or } \\
\text { mobile device } \\
\text { to read online. }\end{array}$ \\
\hline
\end{tabular}

Background: Tumour lysis syndrome is a common complication of haematological malignancies and has historically been managed with hyperhydration, urine alkalinisation and allopurinol with renal dialysis reserved for patients in acute renal failure. Rasburicase has been shown to drastically reduce the need for dialysis; however, its use is limited in developing countries owing to its cost and availability.

Aim: This retrospective analysis aimed to compare the cost to state per patient of rasburicase compared to dialysis in the management and prevention of tumour lysis syndrome in paediatric patients presenting with haematological malignancies admitted to Red Cross War Memorial Children's Hospital (RCWMCH).

Setting: Red Cross War Memorial Children's Hospital.

Methods: Patients from two consecutive 35 month periods, before and after the availability of rasburicase at $\mathrm{RCWMCH}$, were grouped according to treatment modality, and the cumulative costs of hospitalisation, dialysis and drug administration were compared.

Results: The groups were comparable in mean age and gender. The mean total length of hospital stay was 10.04 days shorter for the rasburicase group than the dialysis group with the average cost per patient in the rasburicase group being R40 989.64 lower than the dialysis group.

Conclusion: The use of rasburicase results in a significant per patient cost saving when compared to dialysis, which often requires intensive care admission, and results in extended hospitalisation. The study supports the continued use of rasburicase as an essential adjunct in the management and prevention of tumour lysis syndrome, reaffirming its use as a cost-effective and efficient drug.

\section{Introduction}

Tumour lysis syndrome (TLS) and hyperuricaemia are common complications of haematological malignancies and have historically been managed with hyperhydration, urine alkalinisation and allopurinol, with renal dialysis reserved for patients in acute renal failure. . $2,3,4,5^{\text {The prevention }}$ and management of TLS presents a therapeutic challenge for oncologists treating children with leukaemias and lymphomas in limited-resource settings, owing to cost implications. ${ }^{6}$ Randomised control trials have shown that rasburicase, a recombinant form of urate oxidase, is a superior drug for the management of TLS when compared to allopurinol, and while it forms part of standard treatment protocols in certain countries, its availability within our context is limited owing to cost. ${ }^{7,8}$

Prior to the availability of rasburicase at Red Cross War Memorial Children's Hospital (RCWMCH) in September 2014, all patients with TLS who developed severe electrolyte abnormalities despite medical therapy, worsening renal failure and/or fluid overload required an intensive care unit (ICU) admission and acute haemodialysis as a therapeutic rescue modality. Haemodialysis is costly and requires specialised equipment, personnel and expertise. Rasburicase was preferred on the basis that it would reduce treatment-related morbidity and potentially reduce the reliance on dialysis.

Tumour lysis syndrome does not occur in all patients receiving treatment for haematological malignancies. At the RCWMCH oncology unit, rasburicase is reserved for patients that meet 
TABLE 1: Indications for administration of rasburicase at Red Cross War Memorial Children's Hospital.

\begin{tabular}{|c|c|c|}
\hline Tumour Type & Indication & Description \\
\hline Acute leukaemia & Raised white cell count & Serum white cell count $>100 \times 10^{9} / \mathrm{L}$ \\
\hline \multirow[t]{3}{*}{$\begin{array}{l}\text { Non-Hodgkin } \\
\text { lymphomas/ } \\
\text { leukaemias }\end{array}$} & Bulk disease & $\begin{array}{l}\text { Large abdominal tumours, Burkitt } \\
\text { leukaemia (i.e. bone marrow } \\
\text { involvement }>25 \% \text { ) }\end{array}$ \\
\hline & Raised initial creatinine & $\begin{array}{l}\text { Above the normal reference range } \\
\text { for age }\end{array}$ \\
\hline & Renal infiltration & $\begin{array}{l}\text { Renal infiltration by leukaemia or } \\
\text { lymphoma on routine imaging } \\
\text { (ultrasound, CT, or MRI scans), } \\
\text { usually but not exclusively in the } \\
\text { context of acute renal failure }\end{array}$ \\
\hline $\begin{array}{l}\text { T-cell lymphoblastic } \\
\text { lymphomas }\end{array}$ & Bulk disease & $\begin{array}{l}\text { Large anterior mediastinal masses, } \\
\text { other bulk disease and/or renal } \\
\text { involvement }\end{array}$ \\
\hline
\end{tabular}

$\mathrm{CT}$, computerised tomography; MRI, magnetic resonance imaging.

certain clinical or serological criteria, or those who are deemed to be at high risk of developing TLS (Table 1).

The dose of rasburicase used at RCWMCH is $0.15 \mathrm{mg} / \mathrm{kg} /$ dose $-0.2 \mathrm{mg} / \mathrm{kg} /$ dose, rounded down to the nearest vial size (1.5 mg per vial), prior to the administration of chemotherapy. A single dose is used with the possibility of a second dose being administered depending of the degree of tumour lysis on day two of chemotherapy. However, to date, only single doses have been prescribed largely owing to cost. This dose is shorter than the manufacturer's recommended dosing of $0.2 \mathrm{mg} / \mathrm{kg}$ daily for 5 days but has been found to be sufficient in preventing TLS in other settings. ${ }^{9}$ Yu et al. conducted a meta-analysis of four relevant studies and found that a single dose of $1.5 \mathrm{mg}$ or $0.15 \mathrm{mg} / \mathrm{kg}$ weight-based single dose is as efficient as the manufacturer's suggested dosing over 5 days and is associated with a $50 \%$ reduction in cost. ${ }^{6,9}$

Rasburicase decreases serum uric acid by $86 \% 4 \mathrm{~h}$ after administration and early use has been shown to decrease the incidence and severity of TLS-induced acute kidney injury, a common indication for dialysis. ${ }^{10}$ Numerous studies have reported a significant reduction in the incidence of paediatric patients with TLS requiring dialysis after being treated with rasburicase. ${ }^{11}$ Unlike allopurinol, rasburicase requires no dosage adjustments for renal function, has no clinically relevant drug-drug interactions, has a rapid onset of action and reduces the pool of pre-formed uric acid within hours. ${ }^{6,8,12}$ It has subsequently become the first-line treatment, in place of allopurinol, in the prevention and management of TLS in paediatric patients in both the USA and Canada with little or no need for dialysis. ${ }^{8}$

The main obstacle preventing widespread use of rasburicase, particularly in limited-resource settings such as ours, is its cost. ${ }^{1,13}$ Numerous studies have assessed the cost-effectiveness of rasburicase compared to allopurinol. These have shown that patients treated with rasburicase have significantly greater reductions in plasma uric acid levels, shorter admissions to ICU, shorter overall hospital admissions, as well as markedly reduced incidence of patients requiring renal dialysis. Dialysis has been found to result in a doubling in the length of hospital stay and a tripling of the overall cost of treatment. ${ }^{1,2,14}$ This translates into a significant reduction in the cost of treatment per patient, with Cairo et al. reporting that from a health systems perspective, use of rasburicase in place of allopurinol may in fact be cost saving. ${ }^{1}$

Numerous studies have been conducted to assess the effectiveness of rasburicase at doses lower than and for durations shorter than the manufacturer's recommended dose of $0.2 \mathrm{mg} / \mathrm{kg}$ daily for 5 days. ${ }^{15,16,17}$ A strategy that has been shown to be more effective than allopurinol alone involves utilising a single dose of rasburicase prior to commencement of chemotherapy for low- to intermediaterisk patients, with a longer course of rasburicase reserved for patients who develop TLS-associated hyperuricaemia despite best preventative measures. ${ }^{4,15,18}$ These studies found no inferiority in the response rate of single-dose rasburicase compared to 5-day daily dose for both treatment and prophylaxis of TLS-associated hyperuricaemia. ${ }^{15,16,17}$ This approach has subsequently been adopted in developing countries, such as India, with favourable outcomes. ${ }^{6}$

Annemans et al. found that in patients that develop TLS, the cost per patient is 11 times greater than those with hyperuricaemia alone and no TLS. ${ }^{3}$ This is owing to the cost of dialysis and admission to high-care facilities. ${ }^{3}$ Kennedy \& Ajiboye propose that given the high cost of extended hospitalisation and dialysis for patients that develop renal impairment, prescribing the full recommended course of rasburicase in these cases may in fact still result in substantial cost savings. ${ }^{18}$

It is these findings, and their applicability to a resourcelimited public health sector, that prompted our decision to compare the healthcare-related costs of prescribing rasburicase versus dialysis in paediatric patients presenting with haematological malignancies at RCWMCH. The intention of this study was to guide clinical practice and enhance the allocative efficiency of resources.

\section{Methods}

The study is a retrospective analysis of administrative data of paediatric patients with haematological malignancies who required dialysis or rasburicase in the management or prevention of TLS. The target population included all paediatric patients treated for acute leukaemias ( $\mathrm{T}$ - and B-cell lymphoblastic and Burkitt leukaemias) or nonHodgkin lymphomas (including Burkitt lymphoma and T-cell lymphoblastic lymphoma) at RCWMCH oncology unit over the period from October 2011 to July 2017 (70 months) using a convenience sampling method. A time frame of 70 consecutive months from October 2011 to July 2017 was used and divided into two consecutive periods of 35 months: the first prior to the availability of rasburicase (October 2011 to August 2014), when patients were dialysed for TLS, and the second subsequent to the availability of rasburicase (September 2014 to July 2017), where patients at high risk of developing TLS or with established TLS (thus meeting the criteria for dialysis) were managed with rasburicase. 
From the population, the study sample comprised three sub-groups, which included all patients who required haemodialysis for TLS, all patients that received rasburicase for the prevention of TLS and patients that met the criteria for rasburicase (prior to its availability) but were treated with allopurinol and hyperhydration. Patients were excluded from the study if they had a haematological malignancy that did not require dialysis or rasburicase, any histological diagnoses other than acute leukaemia or non-Hodgkin lymphomas, any pre-existing renal impairment prior to diagnosis from a cause not related to their leukaemia or lymphoma or if they required dialysis for any reason other than TLS.

The cost comparison was done from a healthcare service perspective only and did not include costs incurred by patients in their personal capacity. This was in keeping with available literature which have evaluated the cost saving of rasburicase use versus other therapeutic modalities. ${ }^{1,4}$ The amount of rasburicase received, amount of allopurinol received, type and mode of dialysis, length of general hospital ward and ICU admission, as well as serum sodium, potassium, creatinine, urea, calcium, magnesium and phosphate were determined for each patient. Cumulative costs were then determined for each of the two respective time periods, as well as per patient costing between sub-groups. Statistical methods utilised to analyse the data included graphical methods of association (bar graphs), descriptive measures (proportions or percentages) and hypothesis testing (paired $t$-test).

\section{Ethical considerations}

Ethical approval was obtained from the University of Cape Town's Human Rights Ethics Committee (UCT HREC $515 / 2017)$ and all data collection was centralised at RCWMCH oncology unit.

\section{Results}

\section{Sample population}

One hundred and fifty-five patients were diagnosed with biopsy proven haematological malignancies at RCWMCH from October 2011 to July 2017. This comprised 116 patients with acute lymphoblastic leukaemia (ALL), 31 with Burkitt lymphoma/leukaemia (NHL) and eight with T-cell lymphoma (TCL). Of the 155 newly diagnosed patients, six patients were included in the dialysis group and 17 patients in the rasburicase group, while six patients formed the comparator group. The algorithm in Figure 1 refers.

The groups were comparable in mean age and gender (Table 2). The tumour profile differed slightly between the groups with no T-cell lymphomas being observed in the rasburicase group. There was a notable difference in the mean number of days hospitalised and mean days in ICU between the groups. Hyperleukocytosis formed the predominant indication for rasburicase with a mean white

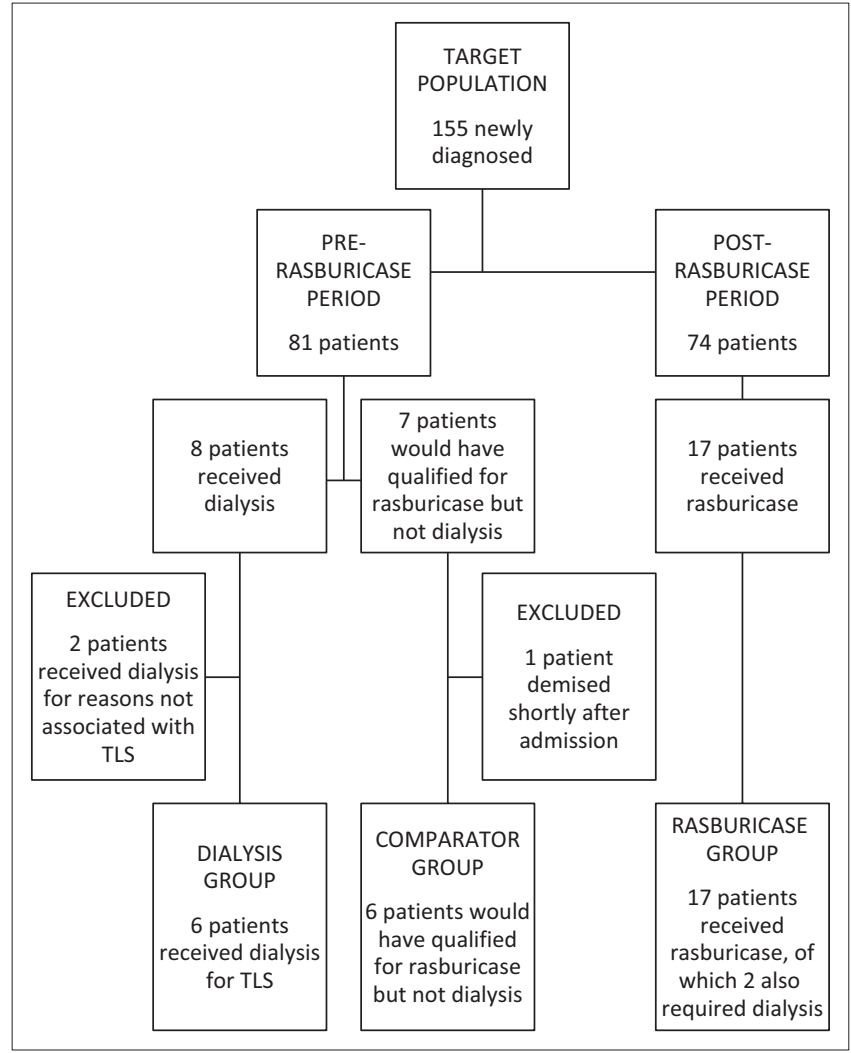

TLS, tumour lysis syndrome.

FIGURE 1: Demographic and clinical characteristics.

TABLE 2: Demographic characteristics of sub-groups.

\begin{tabular}{lccc}
\hline Characteristic & $\begin{array}{c}\text { Dialysis } \\
(\boldsymbol{n}=6)\end{array}$ & $\begin{array}{c}\text { Comparator } \\
(\boldsymbol{n}=6)\end{array}$ & $\begin{array}{c}\text { Rasburicase } \\
(\boldsymbol{n}=\mathbf{1 7})\end{array}$ \\
\hline Mean age, years (SD) & $7.17(1.94)$ & $4.5(3.45)$ & $7.65(5.02)$ \\
Age range, years & $5-10$ & $0-10$ & $1-15$ \\
Female sex, $n$ (\%) & $3(50.00)$ & $3(50.00)$ & $7(41.18)$ \\
On private medical aid, $n(\%)$ & $4(66.67)$ & $4(66.67)$ & $7(41.18)$ \\
Tumour type, $\boldsymbol{n}(\%)$ & & & \\
$\quad$ Acute lymphoblastic leukaemia & $2(33.33)$ & $5(83.33)$ & $11(64.71)$ \\
Burkitt lymphoma & $3(50.00)$ & $1(16.67)$ & $5(29.41)$ \\
$\quad$ T-cell lymphoma & $1(16.67)$ & $0(0.00)$ & $1(5.88)$ \\
Mean days hospitalised, $n$ (SD) & $34.33(17.46)$ & $33(8.24)$ & $24.29(22.66)$ \\
Mean days in ICU, $n$ (SD) & $2.33(1.21)$ & $0(0.00)$ & $0.29(0.77)$ \\
Indication for rasburicase, $\boldsymbol{n}(\%)$ & & & $9(52.94)$ \\
$\quad$ Hyperleukocytosis & Not applicable & $4(66.67)$ & $7(41.18)$ \\
$\quad$ Bulk disease & Not applicable & $2(33.33)$ & $1(5.88)$ \\
Raised initial creatinine & Not applicable & $0(0.00)$ & \\
\hline
\end{tabular}

SD, standard deviation; ICU, intensive care unit.

cell count (WCC) of 204 in the comparator group and a mean WCC of 365 in the rasburicase group. Bulk disease constituted the predominant indication for rasburicase in patients with Burkitt lymphomas. Only one patient qualified for rasburicase based on a raised initial creatinine.

\section{Patients who received both treatment modalities}

Two patients were dialysed despite receiving a single dose of rasburicase and thus received both treatment modalities. Both these patients presented with established renal failure with elevated calcium-phosphate product ratios (with one 
patient having hyperphosphataemia of $4.42 \mathrm{mg} / \mathrm{dL}$ ) putting them at increased risk of nephrocalcinosis. The decision to provide rasburicase was made by the treating oncologist and nephrologist. The costs of these two patients have been included in the rasburicase group, as well as independently compared to the overall outcomes of the cost comparison (Table 5) to provide information on the appropriateness of the allocation of rasburicase.

\section{Length and cost of hospital stay}

The mean total length of hospital stay for the dialysis group was $10.04(43.33 \%)$ days more than the rasburicase group. The two patients that received both treatment modalities were the only two in the rasburicase group that required an ICU admission. The average length of ICU stay for the rasburicase group was thus 0.29 days, while for dialysed patients the average length of ICU stay was 2.33 days with no patients in the comparator group requiring ICU admission. The accommodation cost (per patient per day) to $\mathrm{RCWMCH}$ for admission to ICU is significantly more than in a general medical ward (Table 3). These figures reflect the cost of accommodation only and do not include the cost of medical procedures or interventions required.

The mean cost of hospital stay (general ward and ICU stay) for patients in the rasburicase group amounted to South African rand (R)50 218.62 per patient, while for patients in the dialysis group this amounted to R89 910.67 per patient and R65 934.00 per patient for patients in the comparator group.

\section{Cost of dialysis}

Since November 2015, RCWMCH has simplified their payment structure for dialysis by outsourcing a company to provide service and consumables. Prior to this, the same company provided the service but not the consumables, which had to be bought by RCWMCH. The cost to RCWMCH for acute haemodialysis, continuous venovenous haemodialysis and chronic dialysis, including the respective service fees and cost of consumables, but excluding the haemodialysis line and cost of insertion), differs considerably (Table 3). The cost of the insertion of a haemodialysis line includes the facility fee (R508.00) and surgical/anaesthetic specialist fee (R1036.00) amounting to a total of R1544.00, as well as the cost of the catheter (R4193.50), which is not included in the consumables for the various dialysis modalities mentioned above. Theatre time is not billed for at RCWMCH for the insertion of central venous lines. The cost of the applicable dialysis modality as well as the cost of insertion of the catheter and the cost of the catheter itself are all included in the calculations of total cost of dialysis per patient (Tables $3 \& 4$ ).

The mean number of acute dialysis sessions received in the dialysis group was two sessions per patient. Two patients required continuous veno-venous haemodialysis as a result
TABLE 3: Accommodation and treatment costs at Red Cross War Memorial Children's Hospital.

\begin{tabular}{lc}
\hline Item & Cost \\
\hline Accommodation costs & \\
General ward admission (per day) & $\mathrm{R} 1998.00$ \\
ICU admission (per day) & $\mathrm{R} 9134.00$ \\
Medication costs & \\
Rasburicase (per $1.5 \mathrm{mg}$ vial) & $\mathrm{R} 3495.00$ \\
Allopurinol (per $100 \mathrm{mg}$ tablet) & $\mathrm{R} 0.3271$ \\
Dialysis costs & \\
Acute haemodialysis (per session) & $\mathrm{R} 2389.07$ \\
CVVHD (per day) & $\mathrm{R} 3000.01$ \\
Chronic haemodialysis (per day) & $\mathrm{R} 855.00$ \\
Cost of insertion of dialysis catheter & $\mathrm{R} 1544.00$ \\
Cost of dialysis catheter & $\mathrm{R} 4193.50$ \\
\hline
\end{tabular}

ICU, intensive care unit; CVVHD, continuous veno-venous haemodialysis.

TABLE 4: Mean total cost per patient.

\begin{tabular}{lccc}
\hline Component & \multicolumn{3}{c}{ Cost (R) } \\
\cline { 2 - 4 } & $\begin{array}{c}\text { Dialysis group } \\
(\boldsymbol{n}=\mathbf{6})\end{array}$ & $\begin{array}{c}\text { Comparator } \\
\text { group }(\boldsymbol{n}=\mathbf{6})\end{array}$ & $\begin{array}{c}\text { Rasburicase } \\
\text { group }(\boldsymbol{n}=\mathbf{1 7})\end{array}$ \\
\hline Mean cost of stay in general ward & $\mathrm{R} 68598.00$ & $\mathrm{R} 65934.00$ & $\mathrm{R} 48069.44$ \\
Mean cost of stay in ICU & $\mathrm{R} 21312.67$ & - & $\mathrm{R} 2149.18$ \\
Mean cost of dialysis & $\mathrm{R} 11719.29$ & - & $\mathrm{R} 1377.67$ \\
Mean cost of rasburicase & - & - & $\mathrm{R} 9045.89$ \\
Mean cost of allopurinol & $\mathrm{R} 8.18$ & $\mathrm{R} 6.76$ & $\mathrm{R} 6.32$ \\
\hline Total cost per patient & $\mathrm{R} 101 \mathbf{6 3 8 . 1 4}$ & R65 940.76 & R60 648.50 \\
\hline
\end{tabular}

$\mathrm{R}$, South African rand; ICU, intensive care unit.

of the portable reverse osmosis systems not being available at the required time and not because of a medical indication. No-one required chronic dialysis. This amounted to an average cost of R11 719.29 per patient for dialysis for TLS.

\section{Cost of rasburicase}

Rasburicase became available in September 2014 and at the time of the investigation it cost R3495.00 per $1.5 \mathrm{mg}$ vial. The mean dose of rasburicase received was $3.88 \mathrm{mg}$ per patient. Given RCWMCH oncology unit's practice of rounding the required dose of rasburicase down to the nearest vial $(1.5 \mathrm{mg}$ rasburicase per vial), the mean number of vials required by each patient was 2.59 vials working out to a mean cost of R9045.89 per patient.

\section{Cost comparison}

Over the two time periods assessed, the total cost to RCWMCH for patients managed for TLS in the dialysis group ( $n=6$ ) was R609 828.84, while the total cost of the comparator group $(n=6)$ was R395 644.56. Thus, the total cost for patients that would have met the criteria for rasburicase prior to its availability (dialysis and comparator groups, $n=12$ ) was R1 005 473.40. The total cost for the rasburicase group ( $n=17)$ was R1 019 290.28.

The cost per patient in the dialysis group was R40 989.64, $67.59 \%$ more than the cost per patient in the rasburicase group (see Table 4). A sub-group analysis of the rasburicase group revealed that the per patient cost for the two patients that received both treatment modalities was R11 660.60, 19.67\% more than the cost of patients that received rasburicase 


\begin{tabular}{|c|c|c|c|c|}
\hline \multirow[t]{2}{*}{ Component } & \multicolumn{4}{|c|}{ Cost (R) } \\
\hline & Dialysis group $(n=6)$ & Comparator group $(n=6)$ & Rasburicase without dialysis ( $n=15)$ & Rasburicase and dialysis $(n=2)$ \\
\hline Mean cost of stay in general ward & R68 598.00 & R65 934.00 & R50 882.30 & R26 973.00 \\
\hline Mean cost of stay in ICU & R21 312.67 & & R0.00 & R18 268.00 \\
\hline Mean cost of dialysis & R11 719.29 & & & R11 710.18 \\
\hline Mean cost of rasburicase & & & R8388.00 & R13 980.00 \\
\hline Mean cost of allopurinol & R8.18 & R6.76 & R5.51 & R5.23 \\
\hline Total cost per patient: & R101 638.14 & R65 940.76 & R59 275.81 & R70 936.41 \\
\hline
\end{tabular}

$\mathrm{R}$, South African rand; ICU, intensive care unit.

without requiring dialysis (see Table 5). Although dialysis had not been avoided in these cases, the use of rasburicase shortened the average length of hospital stay by 21 days $(57.73 \%)$ and reduced the average number of dialysis sessions (by $16.67 \%$ ) required to normalise patients' clinical conditions. This resulted in a reduced cost to the hospital for treating these patients. The cost per patient of the comparator group was R5292.26 more than the per patient cost of the rasburicase group. This is because of the length of initial hospital admission being on average 8.71 days $(35.86 \%)$ longer. The cost implications of allopurinol across the sub-groups were negligible owing to the low cost of the drug.

\section{Discussion}

We conducted a retrospective analysis of paediatric patients who either received rasburicase or required dialysis for the management of TLS. Our study found that on average patients who were treated with rasburicase required a shorter length of initial hospital admission and fewer required ICU admissions when compared to patients that required dialysis for TLS. The hospital-related cost for treatment per patient was $67.59 \%$ more and mean length of hospital stay $73.02 \%$ longer in the dialysis group, compared to the rasburicase group. These findings are similar to those of Candrilli et al., who found that renal dialysis resulted in a doubling of the length of hospital stay and a tripling in costs when compared to rasburicase treatment. ${ }^{2}$

Numerous studies have been conducted to compare the economic ramifications of using rasburicase in place of allopurinol, and while the drug itself may be more costly, it results in shorter hospital admissions, quicker resolution of serum electrolyte levels, decreased incidence of paediatric ICU admission or dialysis and an overall lower level of morbidity and risk of mortality. ${ }^{3,7,11}$ Similar results were found in this study with the length of hospital stay for the rasburicase group being significantly shorter (8.71 days) compared to the comparator group, despite both groups initially receiving similar amounts of allopurinol.

\section{Costs to state}

The mean cost for treatment per patient was significantly lower for the rasburicase group, compared to the dialysis and comparator groups and while two patients required both treatment modalities of interest resulting in a higher per patient cost than those receiving rasburicase without dialysis, it was still significantly lower than the per patient cost for patients that were dialysed without rasburicase. This suggests that the use of rasburicase in such instances may have impacted the clinical severity of the TLS and shortened the period of dialysis required and as such resulted in a cost saving in favour of rasburicase. This translates into the total realised cost to state of the rasburicase group (R1 019 290.28) comprising 17 patients being only slightly more than the total realised cost for the 12 patients that would have met the criteria for rasburicase prior to its availability (R1 005 473.40). Considering historically 50\% of patients failed conservative therapy and went on to be dialysed $(6 / 12)$, if we assume that half of the patients in the second cohort (17) would also have needed dialysis in the absence of rasburicase then this more starkly favours the per patient saving in the rasburicase group.

\section{Risks associated with dialysis}

In addition to the costs associated with dialysis, the healthrelated risks of dialysis versus rasburicase also need to be considered. Dialysis requires the insertion of a central venous catheter which requires a general anaesthetic. This carries with it potential associated anaesthetic risks as well as risks of infection (local infection or sepsis) and bleeding. These factors need to be considered when comparing the two treatment modalities as they are associated with prolonged hospital admissions and increased morbidity and mortality. ${ }^{19}$ These risks are abrogated when rasburicase is administered; however, rasburicase is not completely without risk itself and may precipitate haemolytic anaemia in patients with underlying glucose-6-phosphate deficiency (G6PD). ${ }^{20,21}$ As such, this must be interrogated on clinical history prior to its use, especially in families with Mediterranean ancestry. It is a practice at RCWMCH's oncology unit that all male children receiving rasburicase have a G6PD screen done prior to administration of the drug.

\section{Private medical aid}

In an attempt to provide clear insight into the direct cost implications to the RCWMCH oncology unit, we decided not to include the financial implications of having private medical aid on the results. As is the case throughout the public healthcare sector of the country, patients are stratified according to household monthly income, and are required to pay for medical expenses accordingly. While the costs for the various dialysis modalities are considerably higher for patients with private medical aid, the oncology unit bears the costs stipulated in the results regardless of whether the 
patient has medical aid or not. Consequently, calculations in this small study were based on the cost to state for care regardless of household income and whether or not patients had medical aid funding.

\section{Length of hospital stay}

This study only examined accommodation costs associated with admission to general ward and to ICU and did not include the cost of admission into a high-care facility within the oncology unit. This was decided based on the fact that owing to patient burden, the number of high-care patients at any one time may exceed the geographical space designated as a 'high-care' bed within the wards. As such, allocation to a designated geographical high-care space (three beds within RCWMCH's oncology unit) may underestimate the acuity and subsequent billing of many of the patients which is based on access to the designated high-care area. It would thus provide a potentially inaccurate reflection of costs related to care. The exclusion of the cost of high-care admission is also in keeping with available literature looking at economic comparisons of rasburicase use versus other treatment modalities. ${ }^{1}$

\section{Indications for the use of rasburicase}

Guidelines for the management of TLS in children and adults have traditionally used WCC as a discriminator for risk stratification and hence risk for TLS, where a WCC $<50$ was considered low risk, WCC of 50-100 intermediate risk and those $>100$ considered high risk. Historically intermediate-risk groups were assigned allopurinol and hyperhydration compared to the highrisk groups in which the use of rasburicase was recommended..$^{22}$ Other groups have identified serum urate $(>8 \mathrm{mg} / \mathrm{dL}$ ) as being significantly associated with an increased relative risk (RR4.04; $p<0.0001$ ) for developing TLS and renal events (RR10.7; $P<0.00009) .{ }^{23}$ Perhaps future studies should focus on refining the laboratory criteria for the use of rasburicase examining the relationship between total WCC, serum urate and other indicators of tumour burden like serum lactate dehydrogenase.

\section{Limitations}

Our study is limited owing to its retrospective nature, the short time frame between the two periods of assessment and the limited number of patients studied. While every effort was made to avoid the possibility of random error, the small sample size may exaggerate subject variation and compromise the external validity of the results.

\section{Conclusion}

The goal of the study was to provide insight into the financial impact of current rasburicase use at RCWMCH's oncology unit as a way to make cost-effective decisions with regard to the management and prevention of TLS. The current indications used by the oncology unit, despite being developed largely to limit cost and improve clinical outcomes, are in keeping with practices of other resource-limited environments. This study confirms the cost efficiency of rasburicase and reaffirms the decision made by the service to use it in patients meeting the criteria in Table 1 . This has also impacted positively on patient safety.

Cognisant of the difficulty in procuring rasburicase through the section 21 process, which can be arduous and could potentially delay access to this drug, we would strongly advocate for its wider availability across all paediatric oncology units in South Africa as a means to directly impact patient safety (particularly in places where access to paediatric haemodialysis and intensive care services are limited) and also to minimise the growing costs of healthcare interventions in this setting.

\section{Acknowledgements}

The views expressed in this article are our own and do not constitute an official position of the institution.

\section{Competing interests}

The authors declare that they have no financial or personal relationships that may have inappropriately influenced them in writing this article.

\section{Authors' contributions}

M.F.K. conducted the research under the supervision of M.H. A.D., A.v.E., M.M., P.N., M.H. and M.K. all contributed to the writing of the article.

\section{References}

1. Cairo MS, Thompson S, Tangirala K, Eaddy MT. A clinical and economic comparison of rasburicase and allopurinol in the treatment of patients with clinical or laboratory tumor lysis syndrome. Clin Lymphoma Myeloma Leuk. 2017;17(3): 173-178. https://doi.org/10.1016/j.clml.2016.11.003

2. Candrilli S, Bell T, Irish W, Morris E, Goldman S, Cairo M. A comparison of inpatient length of stay and costs among patients with hematologic malignancies (excluding Hodgkin disease) associated with and without acute renal failure. Clin Lymphoma Myeloma Leuk. 2008:8(1):44-51. https://doi. org/10.3816/CLM.2008.n.003

3. Annemans L, Moeremans $K$, Lamotte $M$, et al. Incidence, medical resource utilisation and costs of hyperuricemia and tumour lysis syndrome in patients with acute leukaemia and non-Hodgkin lymphoma in four European countries. Leuk Lymphoma. 2003;44:77-83. https://doi.org/10.1080/1042819021000054661

4. Eaddy M, Seal B, Tangirala K, Davies EH, O'Day K. Economic implications of rasburicase treatment in adult patients with tumour lysis syndrome. Appl Health Econ Health Policy. 2012;10:431-440.

5. Yarpuzlu AA. A review of clinical and laboratory findings and treatment of tumor lysis syndrome. Clin Chim Acta. 2003;333:13-18. https://doi.org/10.1016/S00098981(03)00166-9

6. Latha SM, Krishnaprasadh D, Murugapriya, Scott JX. Single dose rasburicase in the management of tumor lysis syndrome in childhood acute lymphoblastic leukaemia: A case series. Indian K Nephrol. 2015;25(2):91-94. https://doi. org/10.4103/0971-4065.139092

7. Goldman SC, Holcenberg JS, Finklestein JZ, et al. A randomized comparison between rasburicase and allopurinol in children with lymphoma or leukaemia at high risk for tumor lysis. Blood. 2001;97(10):2998-3003. https://doi.org/10.1182/ blood.V97.10.2998

8. Sood AR, Burry LD, Cheng DK. Clarifying the role of rasburicase in tumor lysis syndrome. Pharmacotherapy. 2007;27(1):111-121. https://doi.org/10.1592/ phco.27.1.111

9. Yu X, Liu L, Nie X, et al. The optimal single-dose regimen of rasburicase for management of tumour lysis syndrome in children and adults: A systematic review and meta-analysis. J Clin Pharm Ther. 2017;42:18-26. https://doi.org/10.1111/ jcpt.12479 
10. Husseini A, Sabucedo A, Lamarche J, Courville C, Peguero A. Acute kidney injury associated with tumor lysis syndrome: A paradigm shift. Am J Emerg Med. 2012;30(390):e3-e6.

11. Teo W, Loh T, Tan A. Avoiding dialysis in tumour lysis syndrome: is urate oxidase effective? - A case report and review of literature. Ann Acad Med Singapore. 2007;36(8):679-683

12. Usami E, Kmura M, Iwai M, Teramachi H, Yoshimura T. Analysis of the incidence of tumor lysis syndrome in patients with hematological malignancies treated with rasburicase. Mol Clin Oncol. 2017;6:955-959. https://doi.org/10.3892/ mco.2017.1232

13. Jayabose S, Kumar V, Dhanabalan R, Rajan P, Rathnam K, Viswanathan K. Low-dose rasburicase in haematologic malignancies. Indian J Pediatr. 2015;82(5):458-461. https://doi.org/10.1007/s12098-014-1606-1

14. Galardy PJ, Hochberg J, Perkins SL, Harrison L, Goldman S, Cairo MS. Rasburicase in the prevention of laboratory/clinical tumour lysis syndrome in children with advanced mature B-NHL: A Children's Oncology Group report. Br J Haematol. 2013;163:365-372. https://doi.org/10.1111/bjh.12542

15. Fen X, Dong K, Pence S, Inciardi J, Bhutada N. Efficacy and cost of single-dose rasburicase in prevention and treatment of tumor lysis syndrome: A metaanalysis. J Clin Pharm Ther. 2013;38:301-308. https://doi.org/10.1111/jcpt.12061

16. Alakel N, Middeke JM, Schetelig J, Bornhauser M. Prevention and treatment of tumor lysis syndrome and the efficacy and role of rasburicase. Onco Targets Ther. 2017;10:597-605. https://doi.org/10.2147/OTT.S103864
17. Dinnell J, Moore BL, Skiver BM, Bose P. Rasburicase in the management of tumor lysis: An evidence-based review of its place in therapy. Core Evid. 2015; 10:23-38.

18. Kennedy LD, Ajiboye VO. Rasburicase for the prevention and treatment of hyperuricaemia in tumor lysis syndrome. J Oncol Pharm Pract. 2010;16:205-213. https://doi.org/10.1177/1078155209348719

19. Allon M, Radeva M, Bailey J, et al. The spectrum of infection-related morbidity in hospitalized haemodialysis patients. Nephrol Dial Transplant. 2005:20(6): 1180-1186. https://doi.org/10.1093/ndt/gfh729

20. Browning L, Kruse J. Hemolysis and methemoglobinemia secondary to rasburicase administration. Ann Pharmacother. 2005;39:1932-1935. https://doi.org/10.1345/ aph.1G272

21. Bauters T, Mondelaers V, Robays H, De Wilde H, Benoit $Y$, De Moerloose B. Methemoglobinemia and hemolytic anemia after rasburicase administration in a child with leukaemia. Int J Clin Pharm. 2011;33:58-60. https://doi.org/10.1007/ s11096-011-9484-3

22. Coiffier B, Altman $\mathrm{A}$, Pui $\mathrm{CH}$, et al. Guidelines for the management of pediatric and adult tumor lysis syndrome: An evidence-based review. J Clin Oncol. 2008;26:2767-2778. https://doi.org/10.1200/JCO.2007.15.0177

23. Cohen LF, Balow JE, Magrath IT, et al. Acute tumor lysis syndrome: A review of 37 patients with Burkitt's lymphoma. Am J Med. 1980;68:486-491. https://doi. org/10.1016/0002-9343(80)90286-7 